\title{
DATA BASED ONTOLOGY CONSTRUCTION COUPLED TO EXPERT SYSTEM FOR STEAM TURBINE AIDED DIAGNOSTIC
}

\author{
KLAI Sihem ${ }^{1}$ and KHADIR Med Tarek ${ }^{2}$ \\ Departement of Computer Science, University 20 Aout 55, Skikda, Algeria ${ }^{1}$ \\ LabGed, Departement of Computer Science, University of Badji Mokhtar of Annaba, Algeria ${ }^{2}$ \\ klai@labged.net ${ }^{1}$ Khadir@labged.net ${ }^{2}$
}

\begin{abstract}
This paper describes an approach for ontology construction using heterogeneous databases. We propose a mono-ontology of multiple CCOs (Canonical Conceptual Ontology) approach coupled to an expert system. Each database is described by its own CCO. The NCCO (Non Canonical Conceptual Ontology) are defined and used for realising inter-CCO mapping and to express the relationships between the COO. An expert system JESS (Java Expert System Shell) is integrated into ontology to generate automatically the NCCO starting from the logical rules. The proposed approach is applied for designing a fault diagnostic maintenance system for steam turbine. The main data and information constituting the system come from disparate data bases with different usage. In this case a database for equipment characteristics and another containing maintenance acts defining symptoms, defects and remedies for maintenance cases. The second aspect of the paper focuses on the possible enhancement and evolution of the developed ontology in order to take into account new maintenance cases.
\end{abstract}

Ontology Construction and Evolution, Expert System (JESS), DataMaster

1. Introduction

In order to achieve an efficient interoperability between heterogeneous information systems, many solutions have been proposed [1]. Ontologies play an important role in resolving semantic heterogeneity by providing a shared comprehension of a given domain of interest. Wache in [2] proposed three main ontology based approaches : single, multiple and hybrid.

- Single ontology approaches uses one global ontology providing a shared vocabulary for the specification of the semantics. It can be applied to integration problems where all information sources to be integrated provide nearly the same view on a domain. But if one information source has a different view on a domain, becomes a difficult task. Also, single ontology approaches are susceptible for changes in the information sources which can affect the conceptualization of the domain represented in the ontology, these disadvantages led to the development of multiple ontologies approaches.

- Multiple ontologies: each information source is described by its own ontology. The semantic of an information source is described by a separate ontology. The inter- ontology mapping is very difficult to define.

- Hybrid approaches: To overcome the drawbacks of the single or multiple ontology approaches, hybrid approaches similar to multiple ontology approaches, the semantics of each source is described by its own ontology. But in order to make the local ontologies comparable to each other a global shared ontology is built. The advantage of a hybrid approach is that new sources can easily be added without the need of modification. It also supports the acquisition and evolution of ontologies.

According to a comparative analysis presented in [2], the hybrid approach is the most relevant approach because it allows semantic heterogeneity and flexibility. The mono-ontological approach is simple to realize but it cannot be used for semantically heterogeneous data bases. Multiple ontologies and hybrid approaches are confronted to the heterogeneity problem of ontologies [3]. In this case, it becomes necessary to integrate and make mappings interontologies. In this paper, a mono-ontology constructed from multiple CCOs with integration of semantically heterogeneous data bases ensuring at the same time implementation flexibility and simplicity, is possible. Each CCO is associated at a data base source. The definition of the NCCO between concepts and the instances belonging to different $\mathrm{CCO}$ allows mappings between the various CCO. The definition of the NCCO consists in seeking and representing correspondences between the various schemas of the integrated data. It can be made in a manual, semi-automatic or automatic way. An expert system JESS [4] is merged with the ontology to deduce from new relations starting from the existing concepts to represent the 
mappings automatically or in a semi-automatic way. To test the feasibility of the approach suggested, we built a steam turbine domain ontology by integrating two relational data bases with different usage. One relates to the characteristics of the equipment of the steam turbine and the other relates to the various cases of maintenance defining the symptoms, the defects, the causes and the remedies for each case. The objective set in this paper is, on one hand the proposition of a new approach mono-ontology multiple-CCO databases integration, and on the other hand, applying this approach to the construction of steam Turbine ontology by integrating and merging existing databases, then representing a mapping inter-CCO with NCCO definition. Ultimately, the exploitation and evolution of the obtained ontology is assured by some instructions of JESS language and, it is independent from the ontology editor.

The rest of the paper focuses on a definition and a taxonomy of domain ontologies, Section 2. The proposed approach is detailed in Section 3 . The semi automated development of the steam turbine ontology based on proposed approach is detailed in Section 4. Section 5, shows how the ontology may be maintained by evolving in order to suit any new brake down and diagnoses. Finally, conclusion are drawn in section 6.

\section{Notion of ontologies}

Gruber [5] defines the ontology as: "An ontology is an explicit specification of a conceptualization". The type of an ontology is closely related to its conceptualization objects such as: knowledge representation high level, generic, domain and application. In our case the developed ontology is of domain type, as it contains a number of concepts and a certain vocabulary that defines a targeted domain i.e., the steam turbine and its maintenance aspects.

\subsection{A taxonomy of domain ontologies}

Domain ontologies, may be divided into two main categories [6]:

- Linguistic ontologies (LO) with the objective of a multi lingual and conceptual representation. Those ontologies define words or contextual usages of words.

- Conceptual ontologies (CO) representing the domain objects and proprieties. However within the $\mathrm{CO}$, different ontologies may present different characteristics according to the field of application and the ontology model upon which they were constructed. According to the definition and the distinction between the primitive concepts and the defined concepts, [5] the CO category is divided into 2 categories: Canonical Conceptual Ontology (CCO) which contains only the primitive concepts and Non Canonical Conceptual Ontology (NCCO) which contains the primitive and the defined concepts.

\subsection{A layered model for ontology design}

As specified in [7] the previous observations lead to identify some relationships between CCOs, NCCOs and LOs.

- Mappings between CCO might also be defined of equivalence operators of some NCCO;

- $\quad$ NCCO models can use powerful CCO oriented model constructs to define their own primitives concepts;

- Los might define the various meaning of each word of a particular language by reference to a NCCO. This reference would provide a basis for formal and exact reasoning and automatic translation of context-specific terms.

\section{Proposed approach}

We propose a single ontology approaches with multiple CCO for domain ontology construction. Each data base is described by its own CCO. The semantic of an information source is described by a separate CCO. The NCCO are defined and used for realising inter-CCO mapping. The main advantage of this approache is simplicity and flexibility permitting to addition of new sources without the need of modification. It also permits to integrated two or more databases semantically heterogeneous. A diagram of the described approach is given in Figure1. This approach exploits the NCCO capability to define equivalent concepts and thus to integrate several CCO addressing the same domain.

We discus three dimensions of semantic integration research in this paper:

Mapping discovery: Given two CCO, how do we find similarities between them, determine witch concepts and properties represent similar notions? We can use various characteristics of $\mathrm{CCO}$, such as their structures, definitions of concepts, instances of classes to find mappings.

Representation of mappings (NCCO): Given two CCO, how do we represent the mappings between them to enable reasoning with mappings? The representation of the mappings interCOO consists in defining the NCCO which can be the OWL constructor or the equivalence relation defined starting from the logical rules between the concepts belonging to different $\mathrm{CCO}$. We propose, also, to use inference engine to assert the NCCO automatically starting from the logical rules between concepts belonging to different CCO. 


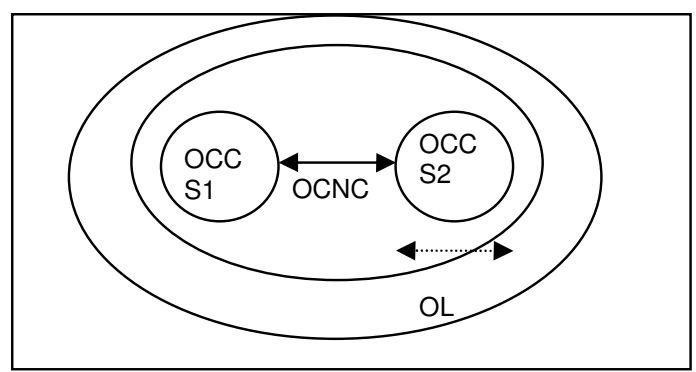

Figure 1: Single ontology approach multiple-CCO

Using Mapping (NCCO): Naturally, defining the mapping between CCO, either automatically or interactively is not a goal in itself. The resulting mappings are used for various integration tasks: to answer the requests users. In this case, this may be realised by using the JESS instructions language.

\section{Steam turbine ontology}

Steam turbines are mechanical devices using supper-heated steam power, and converts it into useful mechanical work. In the studied case, the mechanical work produced is used for electrical production.

Ontologies have been used to represent knowledge and help knowledge inference in the industrial field. As an example, the PROTEUS platform [8] adopted an ontological representation with Cased Based Reasoning (CBR) as model solving problems. Another example is shown in [9] where the authors proposed a collaborative environment allowing users (experts and beginners) to share knowledge through a shared ontology for the maintenance of a steam turbine. Another example showing the specification of an ontology as a domain model allowing solutions for various issues in data indexing, data exchange and data integration may be found in [7].

Each data base containing the steam turbine knowledge or information is described by a CCO, for instance CCOS1 and CCOS2 for the first and second data source respectively. The sum of both $\mathrm{CCO}$ becomes the consensual $\mathrm{CCO}$. In other words, all source data bases available, are merged to form a unique domain ontology. The canonical vocabulary of each data base, becomes a sub group of the consensual CCO. The NCCO operators in this case, will be are the OWL constructors, the equivalent relation or the specific relation of the domain to be created between concepts belonging to different CCOs.

\subsection{Steam turbine ontology conceptualisation}

The implementation of the proposed solution is realised using the Protégé Plug-in DaTaMaster [10]. It was developed for importing data base schemes and their contents in Protégé under OWL. DataMaster permits the integration of multiple data bases in a single ontological representation.

The implementation followed the steps below:

- The choice of the connection driver types: Open Data Base Connectivity (ODBC) or Java Databases Connectivity (JDBC) and the data source;

- The selection of a given table activates the visualisation of its content, then the user have the choice of importing the table or not;

- The chosen data base tables are activated and visualised, each table is transferred into one class or sub-class depending on the user's choice;

\subsection{Steam turbine ontology construction}

The insertion of OWL constructors (owl: unionof,...), relations as well as annotations participate in the process of semantically enhance data belong to different CCO as well as to solves syntaxes and semantic heterogeneity of integrated systems, improving data exchange between them.

Conflict context, was resolved by assigning a unique space name to each CCO. Thus, all the classes, attributes as well as instances belonging to one CCO are pre-tagged with the same Uniform Resource Identifier (URI).

Name conflict was resolved by creating relations. As an example, two classes issued from two different data sources (different $\mathrm{CCO}$ ), where the first one describes the equipment ( code, designation, function, zone,...) and the second details different maintenance cases (code, defects, causes, symptoms and remedies). Both classes treat the same equipment, but are not structured in the same manner and the semantic of their date are different. A relation 
"equivalent1" was created between the two classes and the same time between both instances, Figure 2 .

This method is manual and may be long for a large ontology. Automatic definition of the NCCO is implemented with JESS instructions, then we can discover that the code is a common attribute, thus we can carry out the following algorithm:

For each instance of $\mathrm{CCO} 1$ and for each instance of $\mathrm{CCO} 2$;

If instance.CCO1.code = instance.CCO2.code then

Assert (create in JESS) property "equivalent1" between instance.CCO1 and instance.CCO2

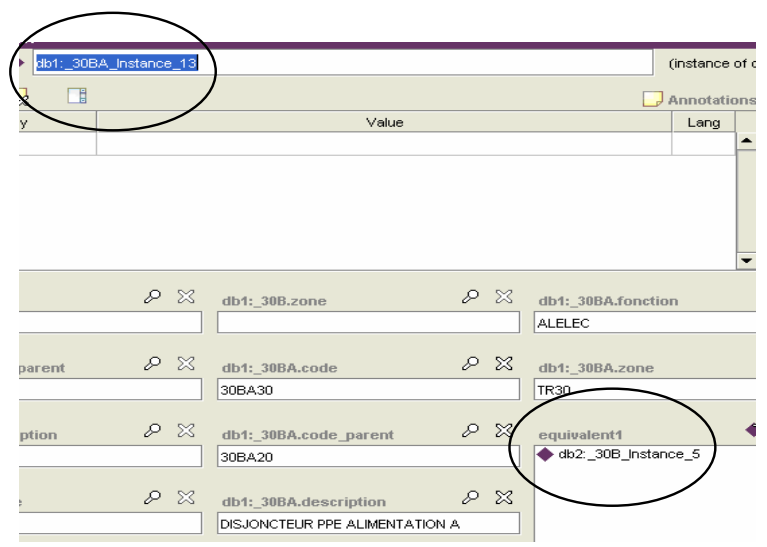

Figure 2: Definition of NCCO with OWL constructors

The hierarchical structure of the steam turbine ontology is shown in Figure 3.

\subsection{Steam turbine Ontology operations}

JESS, is a rule based reasoning engine that can be used with the ontology instances. The OWL2JESS tool [11], permits the conversion of the OWL ontology code to JESS facts. The semantic predefined rules RDFS and OWL are used to verify the coherence and uniformity of the ontology [12]. This will permit to designed, evaluates and refine the original obtained ontology. The obtained knowledge base encapsulates three layers of knowledge: the ontology model layer, the ontology layer and instances layer. The instance layer is represented in Figure 4 , the detail of the others layers are discussed in [13]. The JEES facts are of triplets type given by (Predicate, Subject, Object).

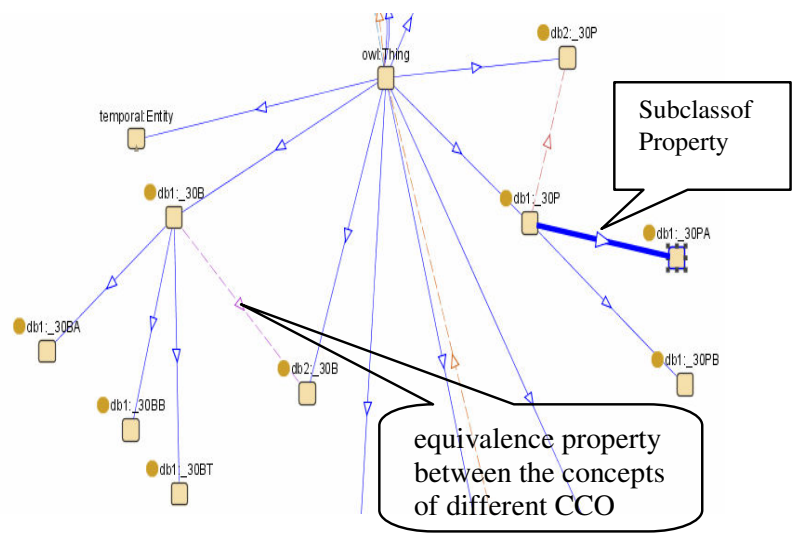

Figure 3: hierarchical structure of Steam turbine ontology

\subsection{Reasoning with mapping using JESS}

The exploitation of the ontology is ensured using rules and requests permitting to fetch the knowledge base through a set of JESS commands such as "defrule" and "defquery". Below an example of using such commands to display the instances equivalent with the instances of the class topo:30BB prefixed with its URI.

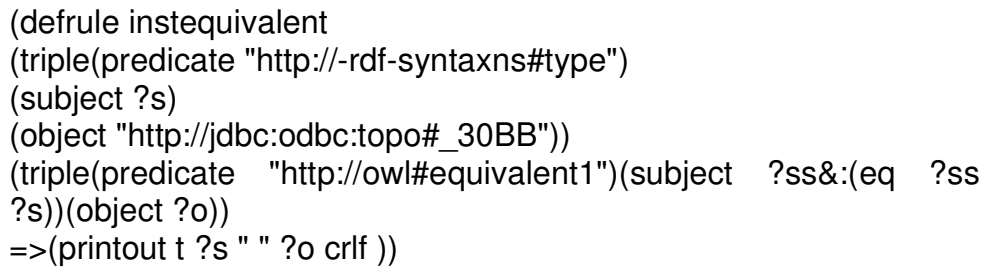




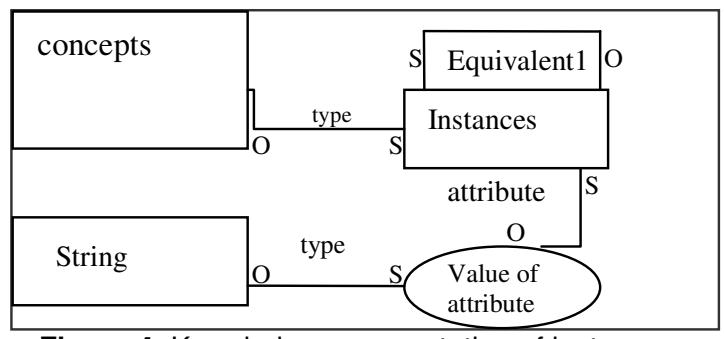

Figure 4: Knowledge representation of instances

\section{Ontology evolution}

In the ontology cycle, ontology evolution is a crucial aspect that needs to be addressed. Ontology evolution is defined as the process of updating the previous ontology version, in order to take into consideration different evolvement of the domain in its conceptualization or application. The evolution process creates a version $n+1$ from the original version $n$. The process is not straight forward and may not be completed manually, as the uniformity and coherence of the ontology must be respected.

\subsection{Methodology}

There are no mature explicit methodology for ontology evolution. However steps can be found in order to elaborate an ontology evolution strategy, given generally by:

- Identification of the domain changes that occurred. There are two main changes identification methods:

Descending identification: Those are imposed by the definition or domain update or the update of the ontology or instances usage.

Ascending identification: Those are changes identified from the ontology analysis it self. For example, by using heuristic rules for ontology optimization.

- Editing ontology changes, that may be complex or elementary.

Elementary changes: a non decomposable change given by a suppression or adding of ontological entities [14].

Complex changes: composed or 2 or more elementary changes that forms together a logical entity [15], e.g., the fusion of a number of concepts in one.

The addition of a new concept must be followed by the insertion of links belongings to the concept along with other existing concepts. The suppression of a concept might cause the isolation of one other concept or more. All conceptual or semantic relations, linking the suppressed concept with other concepts must then be deleted along with linked instances.

- Checking changes made: This step investigates the effects of the changes made on the ontology coherence.

- Validation of changes: changes must be validated, especially in the case of concept fusion or suppression.

- Changes implementation: This must ensure the archiving of the previous version and the preservation of changes made, and metadata associated at the end of this step. A new version of the ontology $n+1$ is created.

- Analysing the compatibility between the version $n$ and $n+1$. This step identifies incompatibilities caused by changes as well as the effects of changes on the compatibilities of version $n+1$ in terms of preservation of the ontology roles.

- Validation of the version $n+1$ in the community: this constitute of the collective validation of the new version by the community of practice.

\subsection{Evolution of the steam turbine ontology}

The operational steam turbine ontology is saved as a text file (knowledge base) on the forma of a triplet set (Predicate, Subject, Object) presented earlier in section

The evolution of this base consists in the elaboration of the addition, suppression and modification operations on the knowledge base using the JESS language "assert" for addition, "modify" for modification, "retract" for suppression. The development of a system which takes in charge the propagation of changes automatically is compulsory. In addition the system must guide the user during the operation in order to make changes in a transparent manner. The original ontology may be saved under a name showing the version and the process of the changes made. This way, the history of the different versions of the ontology may be saved. The change may be implemented on different levels:

- On the ontological level: Adding, suppressing and changing a concept, an attribute, a relation or a semantic relation and updating conceptual relations. 
- On the instances level: Adding, suppressing and changing an instance. The knowledge representation of instances is given in figure 4 .

\subsection{Examples of ontology evolution on the steam turbine}

- Adding an instance and the changes induced. The JESS instruction for changes at a conceptual level for concepts adding and induced changes presented in [16]. Here are the JESS instructions for changes at an instances level:

- The user selects the concepts "30BB" and writes the instances to be created:

(assert (triple

(Predicate "http://www.w3.org/1999/02/22-rdf-syntax-ns\#type")

(Subject

"http://biostorm.stanford.edu/db_table_classes?DSN=jdbc:odbc:diagnostic\#_30BB_Instance_40 ")

(object "http://biostorm.stanford.edu/db_table_classes?DSN=jdbc:odbc:diagnostic\#_30BB")

)

- A set of attributes is displayed, the user chooses an attribute and insert a value of this attribute:

(assert

(triple

(predicate

"http://biostorm.stanford.edu/db_table_classes?DSN=jdbc:odbc:diagnostic\#_30BB.DESCRIPTI

ON-Symptôme")

(subject

"http://biostorm.stanford.edu/db_table_classes?DSN=jdbc:odbc:diagnostic\#_30BB_Instance_1"

) (object "Le Disjocnteur ne se ferme pas")))

- The user selects a relation «equivalent1", a set of instances appurtenant with the other $\mathrm{CCO}$ is displayed, the user chooses an equivalent instance:

(assert (triple

(predicate "http://www.owl-ontologies.com/Ontology1214680546.owl\#equivalent1") (subject "http://biostorm.stanford.edu/db_table_classes?DSN=jdbc:odbc:topo\#_30BB_Instance_4") (object

"http://biostorm.stanford.edu/db_table_classes?DSN=jdbc:odbc:diagnostic\#_30B_Instance_40") ))

\section{CONCLUSION}

A domain ontology is a very efficient approach that permits very efficient knowledge management, giving a unified conceptualisation of the domain. In this paper an ontology with multiple CCOs based on knowledge extraction from two different data sources, which enhanced semantically the final result was presented. The semi-automatic ontology construction helped greatly in terms of time and effort saving. It allowed a faster and more efficient construction than a manual time consuming one. In order to define automatically the NCCO from the logical rules, an expert system JESS is integrated. A strategy for ontology evolution at a conceptual, relational and instances levels are presented in order to update the knowledge base for better diagnoses and maintenance.

\section{Reference}

[1] Amit P. Sheth, Changing Focus on interoperability in information (1999) Interoperating geographic information systems.

[2] Wache H., Vogele T., Visser U., Stuckenschmidt, H., Schuster, G. Neumann, H., and Hubner, S. (2001), Ontology-Based Integration of Information- A Survey of Existing Approaches. In Stuckenschmidt, H., editor, IJCAI-2001 Workshop on Ontologies and Information Sharing.

[3] Klein M., Combining and relating ontologies: an analysis of problems and solutions. In IJCAI-2001 Workshop on Ontologies and Information Sharing, pages 53-62, Seattle, Wa, 2001.

[4] Friedman-Hill, E. (2003), JESS in action : Rule-Based system in Java, Manning publications co., ISBN:1930110893, pp. 480.

[5] Gruber, T.R. (1993), translation approach to portable ontology specifications. Knowledge Acquisit, 5(2), 1993.

[6] Cullot, N., Parent, C., Spaccapietra, S., and Vangenot, C., (2003), Ontologies: A contribution to the Ist International Workshop on semantic Web and Databases (SWDB'03), pages 109-129.

[7] Jean, S., Pierra, G., Ait Ameur, Y., (2007), A Domain Ontologies : A Database-Oriented Analysis. In Web Information Systems and Technologies, International Conferences, WEBIST 2005 and WEBIST 2006, Revised Selected Papers, Lecture Notes in Business Information Processing, pages 238-254, Springer Berlin Heidelberg.

[8] Rasovska, I., Chebel-Morello, B., Zerhouni, N., (2005) Process of s-maintenance: decision support system for maintenance intervention, Emerging Technologies and Factory Automation, ETFA 2005, $10^{\text {th }}$ IEEE, pp.8-15. 
[9] Khadir, M-T, and Sellami, M., (2008), A Web-based Collaborative Environment Based on a Shared Ontology for the Maintenance of Steam Turbines. In 7th Computer Information Systems and Industrial Management Applications, Ostrava, The Czech Republic, June 26 - June 28 , pp151-152.

[10] Nyulas, C., O'Connor, M., Samson Tu, (2007) DataMaster - a Plug-in for Importing Schemas and Data from Relational Databases into Protégé Stanford Medical Informatics ,Stanford University School of Medicine, Stanford, CA 94305.

[11] Mei, J., Bontas, E.P., and Lin, Z., (2005), OWL2Jess: A Transformational Implementation of the OWL Semantics. Lecture notes in computer science, Springer-Verlag, N 3759, pp. 599608.

[12] Mei, J., (2005), Transformation Implementation of OWL Semantic, http://www.ag nbi.de/research/owltrans/

[13] Khadir M.T., Klai S., Aweb-Based Fault Diagnostic Maintenance System for steam Turbines Based on a domain Ontology and Expert System, (2009) International Conference on Multimedia Computing and Systems Proceedings IEEE (ICMCS'09) Ouarzazate, Morocco, ISBN: 978-1-4244-3758-0.

[14] Stanjanovic, L., (2004). Methods and Tools for Ontology Evolution. Thèse de doctorat de l'université de Karlsruhne.

[15] Giorgos F., Dimitris P., Grigoris A., (2006). Evolving Ontology Evolution. Proceedings of the 32nd International Conference on Current Trends in Theory and Practice of Computer science (SOFSEM 06).

[16] Khadir M.T, Klai S., Ontology Construction and Evolution for a steam Turbine Diagnostic Maintenance System, (2009) in International Conference on Recent Advances in Intelligent Information Systems (IIS'09), Krakow, Poland, ISBN 978-83-60434-59-8, pages 399-411. 